\title{
artigo
}

Gomes da Silva, C.F.; Nardi, S.T.; Pinto Neto, J.M.; Ramos, R.R.; Paschoal, V.D.A.;

Fatores de risco para hanseníase identificados a partir de casos notificados num período de 15 anos

\section{Fatores de risco para hanseníase identificados a partir de casos notificados num período de 15 anos}

\author{
Risk factors for leprosy identified from cases notified in a period of 15 years \\ Factores de riesgo de lepra identificados en casos notificados en un periodo de 15 años
}

\begin{abstract}
RESUMO
Objetivo: Identificar e analisar os fatores de risco à transmissão da hanseníase num período de 15 anos. Métodos: Estudo quantitativo, observacional, de corte histórica, histórica e observacional. Dados extraídos do SINAN. As análises estatísticas foram método de U de Mann-Whitney e correlação de spearman, além de análises univariadas. Resultados: No período foram notificados 514 casos. Na análise univariada, os homens chegam ao serviço para serem diagnosticados com maior número de lesões (valor-p<0,05), com as formas clínicas dimorfa e virchowiana, (valor- $p<0,05$ ) e com Grau de incapacidades 1 e 2 (valor- $p<0,05$ ). No modelo final de análise multivariada realizada por meio de regressão logística, encontrou-se significância para associação entre presença de deficiências físicas e forma clínica multibacilar, idade maior de 52 anos e mais de 3 lesões no momento do diagnóstico. Conclusão: 0 sexo e a idade possuem maior risco de infecção à hanseníase. Fatores de riscos sociodemográficos não demonstraram significância nas variáveis clínicas.
\end{abstract}

DESCRITORES: Fatores de risco; Determinantes Sociais da Saúde; Hanseníase; Epidemiologia.

\section{ABSTRACT}

Objective: To identify and analyze risk factors for leprosy transmission over a 15-year period. Methods: Quantitative study, historical and observational. Data extracted from the SINAN. Statistical analyzes were the Mann-Whitney U method and spearman correlation in addition to univariate analyzes. Results: During the period, 514 cases were reported, In the univariate analysis model, men arrive at the service to be diagnosed with a greater number of injuries ( $p$-value $<0.05$ ), with the clinical forms dimorphic and virchovian, ( $p$-value $<0.05$ ) and with Degree of disabilities 1 and 2 ( $p$-value $<0.05$ ). In the final model of multivariate analysis performed by means of logistic regression, significance was found for the association between the presence of physical disabilities and being diagnosed with multibacillaries, being over 52 years old and more than 3 injuries at the time of diagnosis. Conclusion: Sex and age have a higher risk of infection with leprosy. Socio-demographic risk factors did not show significance in the clinical variables.

DESCRIPTORS: Risk factors; Social Determinants of Health; Hansen's disease; Epidemiology.

\section{RESUMEN}

Objetivo: Identificar y analizar los factores de riesgo de transmisión de la lepra durante un período de 15 años. Métodos: Estudio cuantitativo, histórico y observacional. Datos extraídos del SINAN. Los análisis estadísticos fueron el método U de Mann-Whitney y la correlación de Spearman, además de los análisis univariados. Resultados: Durante el período se notificaron 514 casos. En el análisis univariante, los hombres llegan al servicio para ser diagnosticados con un mayor número de lesiones (valor $\mathrm{p}$ $<0,05$ ), con las formas clínicas dimórficas y virchowianas, (valor $p<0,05$ ) y con Grado de discapacidad 1 y 2 (valor de $p<0,05$ ). En el modelo final de análisis multivariado realizado mediante regresión logística, se encontró significancia para la asociación entre la presencia de discapacidad física y forma clínica multibacilar, edad mayor de 52 años y más de 3 lesiones en el momento del diagnóstico. Conclusión: el sexo y la edad tienen un mayor riesgo de infección por lepra. Los factores de riesgo sociodemográficos no mostraron significación en las variables clínicas.

DESCRIPTORES: Factores de Riesgo, Determinantes Sociales de la Salud, Lepra, Epidemiología.

RECEBIDO EM: 30/07/2020 APROVADO EM: 22/09/2020 


\section{Clinton Fábio Gomes da Silva}

Graduado em enfermagem pela FEF, Esp. Em Gestão em saúde e Residência em Vigilância em Saúde. Membro da Rede Brasileira de Enfermagem em Segurança do Paciente, atua no Serviço de Controle de Infecção Hospitalar da FUNFARME/Hospital de Base. ORCID: 0000-0001-8164-2280

\section{Susilene Toneli Nardi}

Graduada em Terapia Ocupacional. Doutorado em Ciências da Saúde pela Faculdade de Medicina de São José do Rio Preto- FAMERP. Pesquisadora Científica VI do CLR - Instituto Adolfo Lutz - São José do Rio Preto-SP. Conselheira Efetiva do CREFITO-3/SP. Membro da Comissão de Ética e Deontologia de Terapia Ocupacional -CEDTO do CREFITO-3. Coordenadora da Câmara Técnica Sócio Sanitária do CREFITO-3. Editora Chefe da Revista Hansenologia Internationalis: hanseníase e outras doenças infecciosas. ORCID: 0000-0001-8793-8437

\section{André Wilian Lozano}

Graduado em enfermagem pela FEF. Mestre em Enfermagem pela Faculdade de Medicina de São José do Rio Preto - FAMERP. Membro da Rede Brasileira de Enfermagem em Segurança do Paciente-REBRAENSP. Professor no curso de Enfermagem da Fundação Educacional de Fernandópolis - FEF e no curso de Medicina da Universidade Brasil.

ORCID: 0000-0002-5721-7054

\section{José Martins Pinto Neto}

Graduado em enfermagem pela FEF. Doutor pelo Programa de Saúde pública da USP. Professor no curso de Enfermagem da Fundação Educacional de Fernandópolis - FEF e no curso de Medicina da Universidade Brasil.

ORCID: 0000-0002-6794-8379

\section{Rogério Rodrigo Ramos}

Graduado em Enfermagem pela FEF. Mestre em Engenharia Biomédica pela Universidade Camilo Castelo Branco. Doutor em Ciências da Saúde pela Faculdade de Medicina de São José do Rio Preto - FAMERP. Professor da Universidade Brasil dos cursos de Medicina e Odontologia. Embaixador na Editora Bentham Science Publishers.

ORCID: 0000-0003-1977-4172

\section{Vania Del'Arco Paschoal}

Graduada em enfermagem pela Universidade do Sagrado Coração, doutorado em Pós Graduação em Ciencias da Saúde pela Faculdade de Medicina de São José do Rio Preto-FAMERP. Docente te na Faculdade de Medicina de São José do Rio Preto-FAMERP. ORCID: 0000-0002-6047-5345

\section{INTRODUÇÃO}

A hanseníase continua sendo um problema de saúde púbica causada pelo Mycobacterium leprae, que é de alta infectividade e baixa patogenicidade, que acomete o sistema nervoso periférico e o tegumento, com uma variedade de sinais de sintomas de acordo com as formas clínicas e polos tendo como uma das principais características as alterações na sensibilidade térmica, dolorosa e tátil. As vias aéreas superiores dos doentes, sobretudo multibacilares não tratados, são a porta de eliminação que também é a mais provável porta de entrada. É uma doença incapacitante, resultando em deficiências físicas especialmente nos olhos, mãos e pés. É uma das doenças de notificação compulsória conforme a Portaria Ministerial n ${ }^{\circ} 1.61$ de 18 de maio de 2020, devido ao impacto na saúde pública. O tratamento é único a partir de setembro de 2020 com a utilização de três drogas: Rifampicina, Dapsona e Clofazimina sendo de 6 meses para os paucibacilares e de 12 meses para os multibacilares de acordo com a Nota Técnica $n^{\circ} 4$ de setembro de $2020^{(1-5)}$.

A Organização Mundial da Saúde (OMS) propôs a eliminação da hanseníase como problema de saúde pública até o ano 2020, ou seja, diagnosticar menos de 1 caso para cada 10.000 habitantes e sem deformidades aparentes ${ }^{(6)}$. No mundo até 2016 reportaram 214.783 casos novos de indivíduos acometidos por hanseníase sendo prevalência de 2,9/100.000, nas Américas houve aumento de $14 \%$ referente a
2015, e o Brasil sobressaiu em primeiro lugar do continente, ocupando o $2^{\circ}$ lugar no mundo, com $13 \%$ dos novos casos (26.395) com a taxa de detecção de 12,2/100.000 habitantes ${ }^{(6,7)}$.

No Estado de São Paulo (ESP) visualiza-se uma queda gradativa do coeficiente de prevalência de 1985 a 2015, estacionando em $0,38 / 10.000$ habitantes, com diminuição na taxa de detecção dos casos novos de 2001 a 2017 , até então em 2,03\% ${ }^{(3,8)}$. O município de São José de Rio Preto (SJRP) mantém a meta de eliminação do agravo desde 2007, quando atingiu menos de um caso por $10 \mathrm{mil} \mathrm{ha-}$ bitantes, mas há oscilações importantes, em 2007 apresentava prevalência de 0,99 casos/10.000 habitantes, em 2009 chegou ao menor parâmetro e apresentou 0,33/10.000 habitantes, 
mas a prevalência foi subindo ano a ano e em 2018 chegou a 0,80/10.000 habitantes ${ }^{(9,10)}$.

Ao analisar o comportamento de uma doença, inevitavelmente analisamos os fatores de riscos e a literatura mostra que as condições de vida e de trabalho estão relacionadas aos diferentes níveis de saúde vivenciados pelas pessoas, a depender da posição social que ocupam na socieda$\mathrm{de}^{(11)}$. Dentre os modelos existentes, destacamos o modelo de Dahlgren e Whitehead, são observados fatores como sexo, idade, estilo de vida, condição de vida e de trabalho dentre outros, e que nos permite visualizar as relações existentes com os diferentes níveis de saúde ${ }^{(12)}$.

A observação do surgimento das enfermidades relacionada a outros fatores, que não somente os biológicos, leva a reflexão sobre porque determinadas populações estão mais sujeitas a adoecer e encontram-se mais vulneráveis a determinadas doenças ${ }^{(13)}$. A essa desigualdade dos níveis de saúde gerada socialmente, que poderia ser evitada por meio de políticas públicas adequadas, dá-se o nome de iniquidades em saúde ${ }^{(11)}$. Monteiro et al. ${ }^{(14)}$ relatam que os "municípios são unidades fundamentais no estudo da reprodução social da doença na qual se materializam as relações culturais e econômicas dos grupos em sua coletividade" e que a análise dos dados em "diferentes contextos espaciais é fundamental para qualificar as distintas especificidades e vulnerabilidades".

Nesse sentido, o trabalho tem como objetivo identificar e analisar os fatores de risco à transmissão da hanseníase em um município do interior do Estado de São Paulo. Considera-se importante conhecê-los para contribuir com o município no (re)planejamento das ações desse Programa para que se possa atingir a meta de eliminação de acordo com o proposto pela Organização Mundial de Saúde ${ }^{(6)}$.
O município, localiza-se no interior do

Estado de São Paulo, à $442 \mathrm{~km}$ da capital, com população estimada de $456.245 \mathrm{mil}$ habitantes em 2018, com área total 431 , $963 \mathrm{~km}$ e densidade demográfica de

945,12 hab./Km2.

Esse município é

considerado uma im-

portante referência

para a região e os es-

tados circunvizinhos

de Minas Gerais e

do Mato Grosso do

Sul, de fácil acesso,

por ser cortada por

linhas ferroviárias,

rodovias estaduais e

federais e possuir um

aeroporto regional.

\section{MÉTODOS}

O desenho desse estudo é do tipo observacional, descritivo, transversal, retrospectivo, corte histórica e quantitativo, que utilizou-se de dados secundários, dos casos de hanseníase, notificados no Sistema de Informação de Agravos de Notificação (SINAN), no período de 15 anos (2001 a 2016) no município de São José do Rio Preto.

O município, localiza-se no interior do Estado de São Paulo, à $442 \mathrm{~km}$ da capital, com população estimada de 456.245 mil habitantes em 2018, com área total $431,963 \mathrm{~km}$ e densidade demográfica de 945,12 hab./Km2. Esse município é considerado uma importante referência para a região e os estados circunvizinhos de Minas Gerais e do Mato Grosso do Sul, de fácil acesso, por ser cortada por linhas ferroviárias, rodovias estaduais e federais e possuir um aeroporto regional ${ }^{(15,16)}$.

O município possui uma distribuição de renda igual a 0,5081, que representa uma distribuição de renda desigual, e um índice de desenvolvimento humano de 0,797, com um índice de Firjan de desenvolvimento municipal de 0,8753 . A taxa de cobertura de esgoto sanitário adequado é de $99 \%{ }^{(15-17)}$.

A população desse estudo refere-se aos casos de hanseníase (Classificação Estatística Internacional - CID 10 de A30.0 a A30.9), que foram diagnosticados no período do estudo. Os dados foram coletados, no período de março a abril de 2018 , por meio do bigdata, do Projeto Hansen da Faculdade de Medicina de São José do Rio Preto (FAMERP), em convênio com Conselho Nacional de Desenvolvimento Científico e Tecnológico, e do SINAN, disponibilizados online pela Secretaria de Estado da Saúde de São Paulo ${ }^{(8,10)}$.

Pela natureza desse estudo não houve necessidade de submetê-lo na Plataforma Brasil para apreciação do Comitê de Ética sendo que houve a aprovação para a utilização dos dados 
Figura 1: Indicadores de monitoramento do progresso de eliminação da hanseníase de São José do Rio Preto, SP, Brasil, de 2001 a 2018. Parâmetros do MS prevalência por 10.000 habitantes: hiper endêmico: $\geq 20,0$; muito alto: 10,0 a 19,9; alto: 5,0 a 9,9; médio: 1,0 a 4,9 e baixo: <1,0. Parâmetros do MS taxa de detecção por 100.000 habitantes: hiper endêmico: >40,0, muito alto: 20,00 a 39,99; alto: 10,00 a 19,99; médio: 2,00 a 9,99; baixo: <2,00.

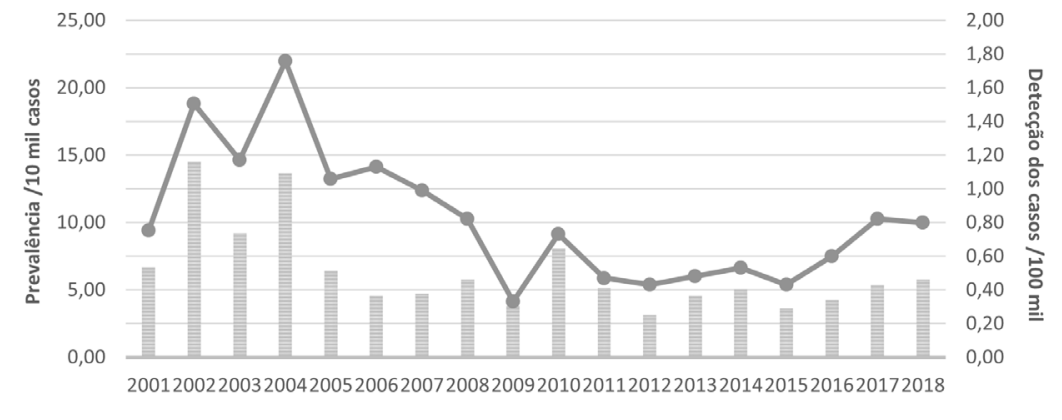

E taxa detecção casos novos/100mil —Taxa de prev/10mil

Fonte: Brasil(3)

Figura 2: Associação entre características clínicas e gênero dos casos de hanseníase notificados em São José do Rio Preto/SP, Brasil, no período de 2001 a 2016.

\begin{tabular}{|c|c|c|c|c|c|c|}
\hline \multirow{2}{*}{ Caracteristicas Clínicas } & \multicolumn{4}{|c|}{ Sexo } & & \\
\hline & \multicolumn{2}{|c|}{ Feminino } & \multicolumn{2}{|c|}{ Masculino } & \multicolumn{2}{|c|}{ TOTAL } \\
\hline & $N$ & $\%$ & $N$ & $\%$ & $N$ & $\%$ \\
\hline Sem Lesão & 74 & 33,64 & 84 & 28,57 & 158 & 30,74 \\
\hline Atéz Lesões & 70 & 31,82 & 54 & 18,37 & 124 & 24,12 \\
\hline$>2$ Lesões & 76 & 34,55 & 156 & 53,06 & 232 & 45,14 \\
\hline TOTAL & 220 & 100,00 & 294 & 100,00 & 514 & 100,00 \\
\hline Nervos Afetados - $p=(0,161)$ & $N$ & $\%$ & $\mathrm{~N}$ & $\%$ & $N$ & $\%$ \\
\hline Sem Nervos Afetados & 66 & 30,00 & 92 & 31,29 & 158 & 30,74 \\
\hline Até 2 Nervos Afetados & 19 & 8,64 & 35 & 11,90 & 54 & 10,51 \\
\hline$>2$ Nervos Afetados & 10 & 4,55 & 26 & 8,84 & 36 & 7,00 \\
\hline Missing & 125 & 56,82 & 141 & 47,96 & 266 & 51,75 \\
\hline TOTAL & 220 & 100,00 & 294 & 100,00 & 514 & 100,00 \\
\hline Forma Clínica $-p=(0,000)$ & $N$ & $\%$ & $N$ & $\%$ & $N$ & $\%$ \\
\hline Indeterminada & 46 & 20,91 & 23 & 7,82 & 69 & 13,42 \\
\hline Tuberculoide & 61 & 27,73 & 35 & 11,90 & 96 & 18,68 \\
\hline Dimorfa & 82 & 37,27 & 144 & 48,98 & 226 & 43,97 \\
\hline Vishoviano & 31 & 14,09 & 92 & 31,29 & 123 & 23,93 \\
\hline TOTAL & 220 & 100,00 & 294 & 100,00 & 514 & 100,00 \\
\hline Grau de incapacidades OMS - $p=(0,002)$ & $N$ & $\%$ & $N$ & $\%$ & $N$ & $\%$ \\
\hline Grau 0 & 137 & 62,27 & 144 & 48,98 & 281 & 54,67 \\
\hline Grau 1 & 58 & 26,36 & 99 & 33,67 & 157 & 30,54 \\
\hline Grau 2 & 19 & 8,64 & 30 & 10,20 & 49 & 9,53 \\
\hline Não Avaliado & 6 & 2,73 & 20 & 6,80 & 26 & 5,06 \\
\hline Missing & 0 & 0,00 & 1 & 0,34 & 1 & 0,19 \\
\hline TOTAL & 220 & 100,00 & 294 & 100,00 & 514 & 100,00 \\
\hline Classe Operacional $-p=(0,000)$ & $N$ & $\%$ & $N$ & $\%$ & $N$ & $\%$ \\
\hline$P B$ & 107 & 48,64 & 58 & 19,73 & 165 & 32,10 \\
\hline$M B$ & 113 & 51,36 & 236 & 80,27 & 349 & 67,90 \\
\hline TOTAL & 220 & 100,00 & 294 & 100,00 & 514 & 100,00 \\
\hline Modo de Entrada $-p=(0,443)$ & $N$ & $\%$ & $N$ & $\%$ & $N$ & $\%$ \\
\hline Caso Novo & 192 & 87,27 & 250 & 85,03 & 442 & 85,99 \\
\hline Transferencia mesmo municipio & 0 & 0,00 & 2 & 0,68 & 2 & 0,39 \\
\hline Transferencia de outro municipio & 7 & 3,18 & 10 & 3,40 & 17 & 3,31 \\
\hline Transferencia de outro Estado & 8 & 3,64 & 8 & 2,72 & 16 & 3,11 \\
\hline Recidiva & 8 & 3,64 & 11 & 3,74 & 19 & 3,70 \\
\hline Outros reingressos & 5 & 2,27 & 13 & 4,42 & 18 & 3,50 \\
\hline TOTAL & 220 & 100,00 & 294 & 100,00 & 514 & 100,00 \\
\hline
\end{tabular}

Fonte: Sistema de Informação de Agravos de Notificação (SINAN). secundários pela FAMERP.

As variáveis analisadas foram as clínica-epidemiológicas: ano e data de notificação, data de diagnóstico, números de lesões, número de nervos afetados, forma clínica da doença, número de avaliações realizadas, classe de operação, modo de entrada e modo de detecção. E as variáveis de determinantes sociais: idade, sexo, gestação, raça, escolaridade, local de moradia.

$\mathrm{Na}$ análise dos dados foram utilizados métodos de Estatística Descritiva e inferenciais, avaliando questões de probabilidades de uma população com base nos dados da amostra para melhor entendimento, foram usados os seguintes métodos, média, mediana, moda, desvio padrão, erro padrão, valor máximo e mínimo, significância, e regressão de Poisson.

Foram feitos testes de hipóteses, usando método de U de Mann-Whitney e correlação de spearman onde analisou em suma, o comportamento das correlações entre as variáveis analisadas e o grau de explicação da variável dependente em relação as variáveis independentes da amostra, além de análises univariadas.

\section{RESULTADOS}

A OMS e o Ministério da Saúde utilizam dois indicadores importantes para avaliar a magnitude e a tendência do agravo, sendo: a taxa de prevalência, calculada sobre o número de casos em curso de tratamento (registro ativo) em determinado local até último dia de cada ano de avaliação, dividido pela população total no mesmo local de tratamento e ano de avaliação multiplicado por $10 \mathrm{mil}^{(2,6)}$. E a taxa de detecção anual de casos novos de hanseníase que é calculada com os casos novos residentes em determinado local e diagnosticados no ano da avaliação (casos novos), dividido pela população total residente, no mesmo local e período, multiplicado por 100 mil habitantes ${ }^{(2)}$. 
$\mathrm{Na}$ figura abaixo, percebe-se que, no município e período estudado, a prevalência com pico de 1,76/10 mil habitantes em 2004 e a detecção com importante aumento no ano 2002, e ambos indicadores com oscilações.

No período 2001 a 2016 foram registrados no SINAN 514 notificações de hanseníase, sendo a média de 32,1 casos/ ano. Em 2004, houve o pico de diagnóstico, com 63 casos (12,26\%), incidência maior entre os homens $(57,2 \%)$, com a faixa etária predominante de 15 - 59 anos $(70,8 \%)$ da cor branca $(71,6 \%)$ com baixa escolaridade, residentes na zona urbana do município (88\%). gicos de 2001 a 2016, a predominância foi para casos multibacilares (MB) (67,9\%), dimorfos (44\%) com menor ou igual a cinco lesões pelo corpo (35,8\%). As avaliações do grau de incapacidade fisica no diágnóstico apresentaram maior incidência de Grau $0(54,7 \%)$ o qual que representa ausência de incapacidade fisica. Em rela-
Quanto aos dados clínicos epidémiolo-

ção ao modo de entrada foram 442 (86\%) de casos novos, 19 (3,7\%) casos de recidivantes, 18 (3,5\%) de outros reingressos; os outros casos (35) vieram de outros municipios, estados ou transferencias dentro do próprio municipio. $\mathrm{O}$ modo de decteção predominou através de encaminhamento $431(83,8 \%)$, demanda espontânea 12 $(2,3 \%)$ e destaque para nove $(1,7 \%)$ casos detectados atráves de exames de contatos, 55 (25\%) fichas de notificações não descreviam esta informação e nove $(4,1 \%)$ apresentaram decteção de outros modos.

No modelo de análise univariada, os resultados mostram que homens chegam ao serviço para serem diagnosticados com maior número de lesões (valor-p $<0,05$ ), com as formas clinicas dimorfa e virchowiana, ou seja $\mathrm{MB}$ (valor-p<0,05) e com Grau de incapacidades 1 e 2 denotando lesão neural e/ou deficiências físicas já instaladas (valor-p<0,05).

A associação entre variáveis sócio demográficas revelou que a escolaridade, a raça e a zona de moradia não interferem nas vari-

\section{Tabela 1. Associação entre características clínicas e idade média dos casos de}

hanseníase notificados em São José do Rio Preto, SP, Brasil, período de 2001 a 2016.

\begin{tabular}{lccc}
\multicolumn{1}{c}{ Variáveis } & Média Idade & Desvio-Padrão & Valor-p \\
Classificação Operacional & & & \\
Paucibacilares ( $\mathrm{n}=165)$ & 46,26 & 16,97 & 0,0191 \\
Multibacilares (n=349) & 50,47 & 15,39 & \\
Grau de incapacidades* & & & \\
Grau O (n=281) & 45,90 & 16,33 & 0,000 \\
Grau 1 (n=157) & 53,19 & 14,18 & \\
Grau 2 (n=49) & 53,59 & 14,54 & \\
*Dados missing. Fonte: Sistema de Informação de Agravos de Notificação (SINAN) &
\end{tabular}

Tabela 2. Variáveis associadas independentemente com deficiências físicas de acordo com a OMS (Graus 1 e 2) de São José do Rio Preto, SP, Brasil de 2001 a 2016.

\begin{tabular}{lccc}
\multicolumn{1}{c}{ Variáveis } & Odds Ratio Ajustado & IC 95\% & P-Value \\
Classificação Operacional (multibacilar) & 1,00 & $0,43-2,30$ & 0,9965 \\
Sexo (masculino) & 1,11 & $0,73-1,68$ & 0,6076 \\
Forma Clínica (dimorfa e virchowiana) & 1,66 & $1,10-2,51$ & 0,0157 \\
Idade (maior que 52 anos) & 1,03 & $1,01-1,04$ & 0,0000 \\
Número de Lesões (mais que três) & 1,04 & $1,01-1,07$ & 0,0019
\end{tabular}

IC - Intervalo de confiança de 95\%; *Análise multivariada por meio de regressão logística não-condicional. Fonte: Sistema de Informação de Agravos de Notificação (SINAN). áveis clínicas como: número de lesões e de nervos afetados, na forma clínica e classificação operacional, e no grau de incapacidades da OMS (valor-p $>0,05)$. A variável idade mostrou que quanto maior a idade, maior o número de lesões encontradas no diagnóstico (valor-p $=0,03$ ), maior a possibilidade de apresentar as formas MB, quer seja, formas clinicas dimorfa e virchowiana da doença (valor-p $=0,01$ ) e de apresentar deficiências físicas (valor-p $=0,00$ ).

No modelo final de análise multivariada realizada por meio de regressão logística, encontrou-se significância para associação entre presença de deficiências físicas e ser diagnosticado nas formas clínicas dimorfa e virchowiana, ter mais de 52 anos e mais de 3 lesões no momento do diagnóstico. A classificação operacional e o sexo não influenciam na presença de deficiências físicas.

\section{DISCUSSÃO}

Estudo brasileiro relata que a queda do indicador de prevalência da hanseníase no Brasil está relacionada às diferenças no desenvolvimento e padrão de vida entre as regiões, inferenciando que a detecção precoce e a redução de incapacidades estão relacionadas à eficiência dos serviços de atenção básica de saúde ${ }^{(18)}$.

A associação entre variáveis sócio demográficas revelou que a escolaridade, a raça e a zona de moradia não interferem nas variáveis clínicas como: número de lesões e de nervos afetados, na forma clínica e classificação operacional, e no grau de incapacidades da OMS. A variável idade demonstra que quanto maior a idade, maior o número de lesões encontradas no diagnóstico, maior a possibilidade de apresentar as formas $\mathrm{MB}$ e de apresentar deficiências físicas. Ao contrário dos achados, pesquisas relatam que a ocorrência do agravo pode estar relacionada a variáveis sociodemográficas, por habitarem em aglomerados e dividirem situações de pobreza, com alto índice de transmissão, uma vez que condições socioeconômicas desfavoráveis e as condições precárias de moradia influenciam o risco de adquirir a doença ${ }^{(19-21)}$. 
Em regiões endêmicas, a faixa etária e as condições de vida foram fatores determinantes para a persistência da doença e aumento da infectividade em uma população pediátrica ${ }^{(19)}$. Nesta investigação os homens chegaram ao serviço para serem diagnosticados com maior número de lesões, classificação MB e com grau de incapacidades 1 e 2, denotando lesão neural e/ou deficiências físicas já instaladas. Este dado foi semelhante a investigação multicêntrica, sobre fatores de riscos de incapacidades em hanseníase na Índia, também considerado um país endêmico, assim como o atraso do paciente aos serviços de saúde e do profissional de saúde em realizar o diagnóstico ${ }^{(22)}$. O diagnóstico tardio continua sendo um dos desafios para o controle das endemias nos países subdesenvolvidos.

$\mathrm{Na}$ analise multivariada realizada por meio de regressão logística, encontrou-se significancia estatística para associação entre presença de deficiências físicas e ser diagnosticado nas formas clínicas dimorfa e virchowiana, ter mais de 52 anos e mais de 3 lesões no momento do diagnóstico. A classificação operacional e o sexo não influenciam na presença de deficiências físicas.

Atualmente, observa-se que embora haja tendência de eliminação da hanseníase em nível nacional, as disparidades regionais resultam na manutenção da doença circulante. E esse estudo vem de encontro com as investigações, visto que nas regiões endêmicas os fatores de riscos são referenciados em sua maioria a condições sociais, econômicas e demográficas ${ }^{(2,18,19,22,23)}$.

Vistos os fatores de riscos, o controle de contatos torna-se um dos pilares estratégicos para realizar a vigilância efetiva. e capacitar os profissionais de saúde para melhorar os registros nos sistemas de saúde, além de otimizar as informações para analisar as causas raízes e trabalhar em foco nos determinantes $^{(24,25)}$. Na tentativa de atingir o plano estratégico da $\mathrm{OMS}^{(6)}$, o município incentiva a política de descentralização para atenção básica, a implantação de ações de controle por meio do Programa de Saúde da Família (PSF), além de educação em saúde para profissionais e população em geral.

Ao longo da última década as pesquisas do comportamento do agravo e ações de prevenção e educação no município foram intensificadas na tentativa de melhorar o perfil epidemiológico, e vêm sendo realizadas com sucesso pelas equipes, o que evidencia um forte com- prometimento de todos os profissionais com a busca por melhorias na perspectiva clínica e epidemiológica do paciente e, por conseguinte, do município.

\section{CONCLUSÃO}

Neste estudo constatou que o sexo (masculino) e a idade (pessoas de mais idade) possuem maior risco de infecção à hanseníase. No período e município estudado, observa-se no montante de notificações a predominância do sexo masculino, de idade média com maior acometimento clínico e físico. Fatores de riscos sociodemográficos não demonstraram significância nas variáveis clínicas.

Para diminuir o risco de exposição evidente, o programa do município deve enfatizar na conscientização da comunidade masculina, com foco em mensagens chaves para atrair o público alvo, estabelecer estratégias de busca ativa de casos e de comunicantes; assim como capacitar, periodicamente, todos os profissionais de saúde para o diagnóstico e o tratamento precoce para quebrar a cadeia de transmissão da doença, além de prevenção das incapacidades físicas (olhos, mãos e pés), do estigma, preconceito e discriminação.

\section{REFERÊNCIAS}

1. Focaccia. R. Tratado de Epidemiologia. $5^{\circ}$ ed. Ribeirão Preto: Atheneu; 2015.

2. Brasil. Ministério da Saúde. Secretaria de Vigilância em Saúde. Departamento de Vigilância das Doenças Transmissíveis. Diretrizes para vigilância, atenção e eliminação da hanseníase como problema de saúde pública. Manual técnico-operacional. Brasília (DF), 2016. Disponível em: http:// www.saude.gov.br/images/pdf/2016/fevereiro/04/diretrizes-eliminacao-hanseniase-4fev16-web.pdf. Acesso em: 15 jul. 2018.

3. Brasil. Ministério da Saúde. Secretaria de Vigilância em Saúde. Caracterização da situação epidemiológica da hanseníase e diferenças por sexo, Brasil, 2012-2016. Boletim Epidemiológico, 2018;49(4):1-12. Disponivel em: https://www. saude.gov.br/images/pdf/2018/janeiro/31/2018-004-Hanseniase-publicacao.pdf. Acesso em: 31 maio 2019.

4. Brasil. Ministério da Saúde. Portaria n 1.061 de 18 de maio de 2020. Revoga a Portaria $n^{\circ} 264$, de 17 de fevereiro de 2020, e altera a Portaria de Consolidação n 4/GM/MS, de 28 de setembro de 2017, para incluir a doença de Chagas crônica, na Lista Nacional de Notificação Compulsória de doenças, agravos e eventos de saúde pública nos serviços de saúde públicos e privados em todo o território nacional. Disponível em: https://www.in.gov.br/en/web/dou/-/portaria-n-1.061-de18-de-maio-de-2020-259143078. Acesso em: 17 set. 2020.

5. Brasil. Ministério da Saúde. Secretaria de Vigilância em Saúde. Departamento de Doenças de Condições Crônicas e Infecções Sexualmente Transmissíveis. Coordenação-Geral de Vigilância das Doenças em Eliminação. Nota Técnica nº 4/2020-CGDE/.DCCI/SVS/MS. Ampliação de uso da clofazimina para hanseníase paucibacilar no âmbito do Sistema Único de Saúde. Disponível em: http://www.aids.gov.br/pt-br/ legislacao/nota-tecnica-no-42020-cgdedccisvsms. Acesso em: 17 set. 2020 . 


\section{REFERÊNCIAS}

6. WHO SEARO; Department of Control of Neglected Tropical Diseases. Global Leprosy Strategy 2016-2020: Accelerating towards a leprosy-free world. 2016, 20 p. Disponivel em: https://www.who.int/lep/resources/9789290225096/en/. Acesso em: 22 fev. 2018.

7. Brasil. Ministério da Saúde. Secretaria de Vigilância em Saúde. Coordenação-Geral de Desenvolvimento da Epidemiologia em Serviços. Guia de Vigilância em Saúde. $3^{\circ}$ ed. Brasília: Ministério da Saúde; 2019. Disponível em: http://portalarquivos2.saude.gov.br/images/pdf/2019/junho/25/guia-vigilancia-saude-volume-unico-3ed.pdf. Acesso em: 11 fev. 2020.

8. São Paulo. Secretária Estadual de Saúde. $1^{\circ}$ Relatório de Monitoramento: Hanseníase. Divisão Técnica de Vigilância Epidemiológica da Hanseníase. Centro de Vigilância Epidemiológica, Coordenadoria de Controle de Doenças. 2016, 47 p. Disponivel em: http://www.saude.sp.gov.br/resources/ cve-centro-de-vigilancia-epidemiologica/areas-de-vigilan$\mathrm{cia} /$ hanseniase/doc/hans16_relatorio2015.pdf. Acesso em: 21 out. 2019.

9. Paschoal VD, Nardi SMT, Cury MRCO, Lombardi C, Virmond $M C L$, Silva RMDN, et al. Criação do banco de dados para sustentação da pós-eliminação em hanseníase. Ciências \& Saúde Coletiva. 2011;16(sup.1);1210-2011. https://doi. org/10.1590/S1413-81232011000700052

10. São Paulo. Secretaria de Estado da Saúde. Divisão Técnica de Vigilância Epidemiológica da Hanseníase/CVE/CCD/ SES. Casos Novos, Coeficiente de Detecção, Prevalência e Coeficiente de Prevalência de Hanseníase distribuídos por GVE/Município de Residência. Estado de São Paulo, 20172018. Disponivel em: http://www.saude.sp.gov.br/resources/ cve-centro-de-vigilancia-epidemiologica/areas-de-vigilancia/hanseniase/dados/hans_cn_ra_serie.pdf. Acesso em: 15 jan. 2019.

11. Fiorati RC, Arcêncio RA, Souza RB. As iniquidades sociais e o acesso à saúde: desafios para a sociedade, desafios para a enfermagem, Rev. Latino-Am. Enfermagem. 2016;24:e2687 https://doi.org/10.1590/1518-8345.0945.2687

12. Buss PM, Pellegrini Filho A. A saúde e seus determinantes sociais. PHYSIS: Rev. Saúde Coletiva. 2007;17(1):77-93. http://dx.doi.org/10.1590/S0103-73312007000100006

13 Simionato De Assis I, Arcoverde MAM, Ramos ACV, Alves LS, Berra TZ, et al. Social determinants, their relationship with leprosy risk and temporal trends in a tri-border region in Latin America. PLOS Neglected Tropical Diseases. 2018;12(4):e0006407. https://doi.org/10.1371/journal. pntd.0006407

14. Monteiro LD, Mota RMS, Martins-Melo FR, Alencar CH, Heukelbach J. Determinantes sociais da hanseníase em um estado hiperendêmico da região Norte do Brasil. Rev Saúde Pública. 2017;51:70. https://doi.org/10.1590/S15188787.2017051006655

15. Ciência, Tecnologia e Inovação. Conjuntura Econômica de São José do Rio Preto. $34^{\circ}$ ed. São José do Rio Preto: Secretaria
Municipal de Planejamento Estratégico, Ciência, Tecnologia e Inovação; 2019. Disponível em: https://www.riopreto.sp.gov. br/wp-content/uploads/arquivosPortalGOV/planejamento/ conjuntura/conjuntura-2019.pdf. Acesso em: 23 mar. 2020.

16. IBGE. Instituto Brasileiro de Geografia e Estatística. Trabalho e Rendimento. São José do Rio Preto. 2017. Disponível em: https://cidades.ibge.gov.br/brasil/sp/sao-jose-do-riopreto/panorama. Acesso em: 11 mar. 2018.

17. SeMAE. Serviço Municipal Autônomo de Água e Esgoto de São José do Rio Preto. Distribuição de renda. 2008. Disponível em: https://semae.riopreto.sp.gov.br/acesso-a-informacao. aspx. Acesso em: 27 nov. 2018.

18. Ribeiro MDA, Silva JCA, Oliveira SB. Estudo epidemiológico da hanseníase no Brasil: reflexão sobre as metas de eliminação. Rev Panam Salud Publica. 2018;42:e42. https://doi. org/10.26633/RPSP.2018.42

19. Rodrigues TSV, Gomes LC, Cortela DCB, Silva EA, Silva $C A L$, Ferreira SMB. Factors associated with leprosy in children contacts of notified adults in an endemic region of Midwest Brazil. J Pediatr. 2019;S0021-7557(18)31082-9. https://doi. org/10.1016/j.jped.2019.04.004

20. Silva EA, Rosa PS, Faria A, Belone F, Maria N, Tomimori J. Serodiagnosis of leprosy and follow-up of household contacts using a commercial rapid test containing ND-O/LID-1 antigens. Lepr Rev. 2017;88(2):174-83. http://dx.doi.org/03057518/17/064053+10

21. Ferreira SMB, Ignotti E, Gamba MA. Fatores associados à reincidência em hanseníase em Mato Grosso. Rev Saúde Pública. 2011;45(4):756-64. https://doi.org/10.1590/S003489102011005000043

22. Srinivas G, Muthuvel T, Lal V, Vaikundanathan K, Schwienhorst-Stich EM, Kasang C. Risk of disability among adult leprosy cases and determinants of delay in diagnosis in five states of India: A case-control study. PLOS Neglected Tropical Diseases. 2019;13(6):e0007495. https://doi.org/10.1371/ journal.pntd.0007495

23. Nery JS, Ramond A, Pescarini JM, Alves A, Strina A, Yurylchihara $M$, et al. Socioeconomic determinants of leprosy new case detection in the 100 Million Brazilian Cohort: a population-based linkage study. Lancet Glob Health 2019;7(9):e1226-36. https://doi.org/10.1016/S2214$109 \times(19) 30260-8$

24. Lozano AW, Pinto Neto JM, Femina LL, Donda P, Silva CGF, Nardi ST, et al. The home as an important leprosy transmission factor. J Nurs UFPE On Line. 2019;13:e241790. https:// doi.org/10.5205/1981-8963.2019.241790

25. da Silva CFG, Nardi SMT, Paschoal VDA. The reliability of information records in the Health Information System: surveillance of intra-domiciliary leprosy contacts. Rev Eletron Comun Inf Inov Saúde. 2019;13(4):831-42. http://dx.doi. org/10.29397/reciis.v13i4.1666 